\title{
Holographic Phase Transitions with Fundamental Matter
}

\author{
David Mateos, ${ }^{1}$ Robert C. Myers, ${ }^{2,3}$ and Rowan M. Thomson ${ }^{2,3}$ \\ ${ }^{1}$ Department of Physics, University of California, Santa Barbara, California 93106-9530, USA \\ ${ }^{2}$ Perimeter Institute for Theoretical Physics, Waterloo, Ontario N2J 2Y5, Canada \\ ${ }^{3}$ Department of Physics, University of Waterloo, Waterloo, Ontario N2L 3G1, Canada
}

(Received 18 May 2006; published 30 August 2006)

\begin{abstract}
The holographic dual of a finite-temperature gauge theory with a small number of flavors typically contains $D$-brane probes in a black hole background. At low temperature, the branes sit outside the black hole and the meson spectrum is discrete and possesses a mass gap. As the temperature increases, the branes approach a critical solution. Eventually, they fall into the horizon and a phase transition occurs. In the new phase, the meson spectrum is continuous and gapless. At large $N_{c}$ and large 't Hooft coupling, we show that this phase transition is always first order. In confining theories with heavy quarks, it occurs above the deconfinement transition for the glue.
\end{abstract}

DOI: 10.1103/PhysRevLett.97.091601

The gauge-gravity correspondence is a powerful tool to study the nonperturbative physics of gauge theories in diverse dimensions. The classical supergravity regime corresponds to the large- $N_{c}$, strong 't Hooft coupling limit of the gauge theory. This allows the study of a large class of theories that share some of the important features of fourdimensional QCD, such as confinement, chiral symmetry breaking, thermal phase transitions, etc. In principle, because of its asymptotic freedom, QCD itself is not in this class. This means that calculations of certain quantitative properties of QCD, such as the detailed mass spectrum, for example, will require going beyond the supergravity approximation. However, this does not exclude the possibility that some aspects of QCD can be studied in this approximation: Some predictions of the gauge-gravity correspondence may be universal enough to apply to QCD, at least in certain regimes. A suggestive recent example is the gaugegravity calculation of the shear viscosity in the hydrodynamic regime of strongly coupled finite-temperature gauge theories. The viscosity/entropy ratio is universal for a large class of gauge theories in the regime described by their gravity duals [1], which, for a high enough temperature, generically contain a black hole horizon. Moreover, this ratio appears to be surprisingly close to that inferred from experiments at the Relativistic Heavy Ion Collider (RHIC) [2]. It is therefore important to investigate other universal features of the gauge-gravity correspondence.

The quarks in QCD transform in the fundamental representation. For a large class of gauge theories, the holographic description of fundamental matter with a small number of flavors $N_{f} \ll N_{c}$ is provided by $N_{f}$ probe branes in the appropriate gravitational background [3]. At sufficiently high temperature $T$, the latter contains a black hole [4]. Here we exhibit some universal features of this system that depend only on these two facts. In particular, we demonstrate the existence of a first order phase transition for the fundamental matter, as follows:
PACS numbers: 11.25.Tq, 11.10.Wx, 11.15.-q, 11.30.Rd

At sufficiently small $T / M_{q}$ (where $M_{q}$ is the quark mass), the brane tension is sufficient to overcome the attraction of the black hole, and, hence, the branes lie outside the horizon in a "Minkowski" embedding - see Fig. 1 and below. At sufficiently large $T / M_{q}$, the gravitational attraction overcomes the brane tension and the branes fall into the horizon, yielding a "black hole" embedding. In between, a limiting, critical solution exists. We will show that the phase diagram in the vicinity of this solution exhibits a self-similar structure. Thermodynamic considerations show that, as the temperature is raised, the probe branes jump discontinuously from a Minkowski to a black hole embedding at some $T=T_{F}$. Hence (in the approximations stated above), there is a first order phase transition exemplified by discontinuities in, e.g., the quark condensate $\langle\bar{\psi} \psi\rangle$ or the entropy density. Various aspects of this transition were noted before in two specific models $[5,6]$. In the following, we show that the (previously unknown) critical behavior and, as a result, the first order transition are essentially universal to all $D p / D q$ systems.

Confining gauge theories with sufficiently heavy quarks are particularly interesting because two distinct phase transitions occur [5]. At $T=T_{D}$, a black hole appears in the gravitational background. Here the gluons and the adjoint matter become deconfined [4], but the branes remain out-

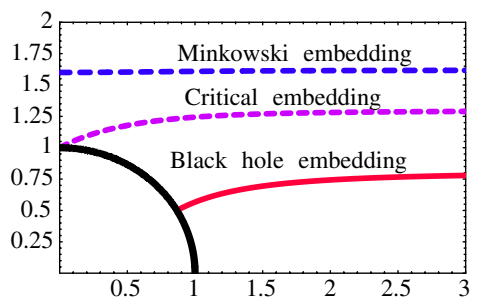

FIG. 1 (color online). Profiles of $D 7$-brane embeddings in a D3-brane background. The thick black circle is the horizon $(\rho=1)$. 
side the horizon. The above transition for the fundamental matter occurs later at $T=T_{F}>T_{D}$.

The string-frame metric for a black $D p$-brane is

$$
d s^{2}=H^{-1 / 2}\left(-f d t^{2}+d x_{p}^{2}\right)+H^{1 / 2}\left(\frac{d u^{2}}{f}+u^{2} d \Omega_{8-p}^{2}\right),
$$

where $H(u)=(L / u)^{7-p}, f(u)=1-\left(u_{0} / u\right)^{7-p}$, and, as usual, $L^{7-p} \simeq g_{s} N_{c} \ell_{s}^{7-p}$, with $\ell_{s}$ the fundamental string length. There is also a nontrivial dilaton $e^{\phi}=g_{s} H^{(3-p) / 4}$ and a Ramond-Ramond field $C_{01 \ldots p}=H^{-1}$. The horizon lies at $u=u_{0}$. Regularity of the Euclidean section, obtained by $t \rightarrow i t_{E}$, requires identifying $t_{E}$ with the period

$$
\frac{1}{T}=\frac{4 \pi L}{7-p}\left(\frac{L}{u_{0}}\right)^{(5-p) / 2} .
$$

String theory on the above background is dual to $(p+1)$ dimensional super-Yang-Mills theory at temperature $T$. In certain cases, one periodically identifies some of the $x_{p}$ directions to produce a lower-dimensional theory at low energies; for example, a $D 4$-brane with one compact direction effectively describes a $(3+1)$-dimensional gauge theory. Under these circumstances, a different background with no black hole may describe the low-temperature physics, and a phase transition may occur as $T$ increases [4]. In the gauge theory, this is a deconfinement phase transition for the gluons and adjoint matter. Below, we assume that $T$ is high enough that the appropriate gravitational background is always Eq. (1).

Now consider a $D q$-brane probe that shares $d$ of the $x_{p}$ directions with the background $D p$-branes and wraps an $S^{n}$ inside the $S^{8-p}$. Assuming that the $D q$-brane also extends along the radial direction, $q=d+n+1$. In the gauge theory, this corresponds to introducing fundamental matter that propagates along a $(d+1)$-dimensional defect. To ensure stability, we assume that the $D p / D q$ intersection under consideration is supersymmetric at zero temperature. Two cases of special interest here are the $D 3 / D 7(n=3)$ [6] and the $D 4 / D 6(n=2)[5]$ systems.

Next, we study the behavior of the brane probe near the horizon, following Ref. [7]. In order to do so, we write

$$
\begin{gathered}
d \Omega_{8-p}^{2}=d \theta^{2}+\sin ^{2} \theta d \Omega_{n}^{2}+\cos ^{2} \theta d \Omega_{7-p-n}^{2}, \\
u=u_{0}+\pi T z^{2}, \quad \theta=\frac{y}{L}\left(\frac{L}{u_{0}}\right)^{(p-3) / 4}, \quad \tilde{x}=\left(\frac{u_{0}}{L}\right)^{(7-p) / 4} x,
\end{gathered}
$$

with $T$ the temperature above. Expanding the metric (1) to lowest order in $z, y$ yields Rindler space together with some spectator directions:

$$
d s^{2}=-(2 \pi T)^{2} z^{2} d t^{2}+d z^{2}+d y^{2}+y^{2} d \Omega_{n}^{2}+d \tilde{x}_{d}^{2}+\cdots,
$$

with the horizon at $z=0$. The $D q$-brane lies at constant values of the omitted coordinates, so they play no role. The $D q$-brane embedding is given by a curve $[z(\sigma), y(\sigma)]$ in the $(z, y)$ plane. Since the dilaton is constant near the horizon, up to an overall constant, the (Euclidean) $D q$-brane action is simply the volume of the brane, i.e., $I \propto$ $\int d \sigma \sqrt{\dot{z}^{2}+\dot{y}^{2}} z y^{n}$, where $\dot{z}=d z / d \sigma$ and $\dot{y}=d y / d \sigma$. Now in the gauge $z=\sigma$, the equation of motion becomes

$$
z y \ddot{y}+(y \dot{y}-n z)\left(1+\dot{y}^{2}\right)=0 .
$$

The solutions fall into two classes: "black hole" and "Minkowski" embeddings (see Fig. 1). Black hole embeddings fall through the horizon and are distinguished by an induced horizon on the brane of size $y_{0}$. The appropriate boundary condition in this case is $d y / d z=0, y=y_{0}$ at $z=0$. Minkowski embeddings are those for which the brane closes off smoothly a distance $z_{0}$ above the horizon. These satisfy the boundary condition $d z / d y=0, z=z_{0}$ at $y=0$. There is also a critical solution, $y=\sqrt{n} z$, which just touches the horizon at $y=z=0$.

Equation (5) enjoys a scaling symmetry: If $y=f(z)$ is a solution, then so is $y=f(\mu z) / \mu$ for any real positive $\mu$. This transformation rescales $z_{0} \rightarrow z_{0} / \mu$ for Minkowski embeddings or $y_{0} \rightarrow y_{0} / \mu$ for black hole embeddings, which implies that all solutions of a given type can be generated from any other one by this transformation.

Consider solutions very close to the critical one $y(z)=$ $\sqrt{n} z+\xi(z)$. Linearizing (5), one finds that for large $z$ the solutions take the form $\xi(z)=z^{\nu_{ \pm}}$, with $\nu_{ \pm}=-n / 2 \pm$ $\sqrt{n^{2}-4(n+1)} / 2$. In the cases of interest (i.e., $d>1$ ), $n \leq 4$ and the exponents are complex, leading to oscillatory behavior, with:

$$
y=\sqrt{n} z+z^{-n / 2}[a \sin (\alpha \log T z)+b \cos (\alpha \log T z)],
$$

where $\alpha=\sqrt{4(n+1)-n^{2}} / 2$ and $a, b$ are constants determined by $z_{0}$ or $y_{0}$. It is easy to show that, under the rescaling discussed above, these constants transform as

$$
\left(\begin{array}{l}
a \\
b
\end{array}\right) \rightarrow \frac{1}{\mu^{(n / 2)+1}}\left(\begin{array}{cc}
\cos (\alpha \log \mu) & \sin (\alpha \log \mu) \\
-\sin (\alpha \log \mu) & \cos (\alpha \log \mu)
\end{array}\right)\left(\begin{array}{l}
a \\
b
\end{array}\right)
$$

This result implies that the solutions exhibit discrete selfsimilarity and yield critical exponents that characterize the near-critical behavior. We refer the reader to Refs. [7,8] for details but emphasize that this behavior is universal for all $D p / D q$ systems with $n \leq 4$.

Each near-horizon solution gives rise to a "global" solution when extended to the full spacetime. The latter are characterized by a quark mass $M_{q}$ and a quark condensate $\langle\bar{\psi} \psi\rangle$, which are read off from the asymptotic behavior. Both of these quantities are fixed by $z_{0}$ or $y_{0}$. As we will see, the values corresponding to the critical solution, $M_{q}^{*}$ and $\langle\bar{\psi} \psi\rangle^{*}$, give a rough estimate of the point at which the phase transition occurs.

To study the global solutions, we introduce an isotropic, dimensionless radial coordinate $\rho$ with

$$
\left(u_{0} \rho\right)^{(7-p) / 2}=u^{(7-p) / 2}+\sqrt{u^{7-p}-u_{0}^{7-p}} .
$$


Now the horizon is at $\rho=1$. For concreteness, we assume that the $D p / D q$ system under consideration is $T$-dual to the $D 3 / D 7$ one, so $(p-d)+(n+1)=4$. Then the Euclidean action density of $N_{f}$ coincident $D q$-branes in the background (1) is

$$
\begin{aligned}
I_{D q}= & \mathcal{N} \int_{\rho_{\min }}^{\infty} d \rho\left(\frac{u}{u_{0} \rho}\right)^{d-3}\left(1-\frac{1}{\rho^{2(7-p)}}\right) \\
& \times \rho^{n}\left(1-\chi^{2}\right)^{(n-1) / 2} \sqrt{1-\chi^{2}+\rho^{2} \dot{\chi}^{2}},
\end{aligned}
$$

where $\mathcal{N}=N_{f} T_{D q} u_{0}^{n+1} \Omega_{n} / 4 T$ is a normalization constant; $T_{D q}=1 /\left(2 \pi \ell_{s}\right)^{q} g_{s} \ell_{s}$, the $D q$-brane tension; $\Omega_{n}$, the volume of a unit $n$ sphere; $\chi=\cos \theta$; and $\dot{\chi}=d \chi / d \rho$.

The equation for $\chi$ derived from (9) yields the large- $\rho$ behavior: $\chi=m / \rho+c / \rho^{n}+\cdots$ [9]. The constants $m$ and $c$ give the quark mass and condensate, respectively, through $[5,6]$

$$
\begin{aligned}
M_{q} & =\frac{u_{0} m}{2^{(9-p) /(7-p)} \pi \ell_{s}^{2}}, \\
\langle\bar{\psi} \psi\rangle & =-\frac{2 \pi \ell_{s}^{2}(n-1) \Omega_{n} T_{D q} u_{0}^{n} c}{4^{n /(7-p)}} .
\end{aligned}
$$

Combining the first with (2) yields the relation $m^{(5-p) / 2}=$ $\bar{M} / T$ between the dimensionless quantity $m$, the temperature $T$, and the mass scale

$$
\bar{M}=\frac{7-p}{2^{(9-p) /(7-p)} \pi L}\left(\frac{2 \pi \ell_{s}^{2} M_{q}}{L}\right)^{(5-p) / 2} \sim \frac{M_{q}}{g_{\mathrm{eff}}\left(M_{q}\right)} .
$$

Here $g_{\text {eff }}^{2}\left(M_{q}\right)=\lambda M_{q}^{p-3}$ is the (dimensionless) effective coupling of the $(p+1)$-dimensional gauge theory evaluated at the quark mass scale [10]. We have also used $\lambda=$ $g_{\mathrm{YM}}^{2} N_{c}$ and $g_{\mathrm{YM}}^{2} \sim g_{s} \ell_{s}^{p-3} \cdot \bar{M}$ is precisely the mass gap in the meson spectrum at temperatures well below the phase transition $[5,11,12]$. Here we see that it is also the scale of the phase transition for the fundamental matter $T_{F} \sim \bar{M}$, since the latter takes place at $m \sim 1$.

A key observation [8] is that for a near-critical solution the values $(m, c)$ are linearly related to the constants $(a, b)$ in the near-horizon region. Together with the transformation rule (7) for the latter and eliminating $\mu$, we deduce that $\left(m-m^{*}\right) / z_{0}^{1+(n / 2)}$ and $\left(c-c^{*}\right) / z_{0}^{1+(n / 2)}$ are periodic functions of $(\alpha / 2 \pi) \log z_{0}$ with unit period for Minkowski embeddings. The same applies for black hole embeddings with $z_{0}$ replaced by $y_{0}$. We have confirmed this behavior numerically.

The oscillatory behavior of $m$ and $c$ as functions of $z_{0}$ or $y_{0}$ implies that the quark condensate is not a single-valued function of the quark mass. The preferred solution will, of course, be the one that minimizes the free-energy density of the $D q$-brane $F=T I_{D q}$. The expression (9) for the latter is divergent, as can be seen by inserting the asymptotic $\chi$ behavior. This divergence must be first regulated by replacing the upper limit of integration by a finite ultraviolet cutoff $\rho_{\max }$. Then it may be renormalized by subtracting the free energy of a fiducial embedding, as in Ref. [5].
Alternatively, one has holographic renormalization [13] for brane probes [14] where a boundary "counterterm" is added to the action (9). For D7-branes in a $D 3$ background, this boundary term is

$$
I_{\text {bound }}=-\frac{\mathcal{N}}{4}\left(\left(\rho_{\text {max }}^{2}-m^{2}\right)^{2}-4 m c\right) .
$$

The renormalized action $I=I_{D q}+I_{\text {bound }}$ is then finite as the cutoff is removed, $\rho_{\max } \rightarrow \infty$. The results for the $D 3 / D 7$ case are shown in Fig. 2 . The plot of the condensate shows the transition is discontinuous, jumping between a Minkowski (point A) and a black hole (point B) embedding. We emphasize again that this first order transition is a direct consequence of the multivalued nature of the physical quantities brought on by the critical behavior described earlier. The transition occurs away from the critical solution and so accessing the self-similar region requires supercooling the system.

It follows from our analysis that the free-energy density takes the form $F=\mathcal{N} T f\left(m^{2}\right)$, where the function $f$ depends only on even powers of $m$ because of the reflection symmetry $\chi \rightarrow-\chi$. Converting the result to field theoretic quantities, the free-energy density scales as

$$
F \sim N_{f} N_{c} T^{d+1} g_{\text {eff }}(T)^{2(d-1) /(5-p)},
$$

where $g_{\text {eff }}^{2}(T)=\lambda T^{p-3}$. The explicit temperature dependence above is that expected on dimensional grounds for a $d$-dimensional defect, and the $N_{f} N_{c}$ dependence follows from large- $N$ counting rules. However, the dependence on the effective coupling is a prediction of the gauge-gravity correspondence for strong coupling. It would be interesting
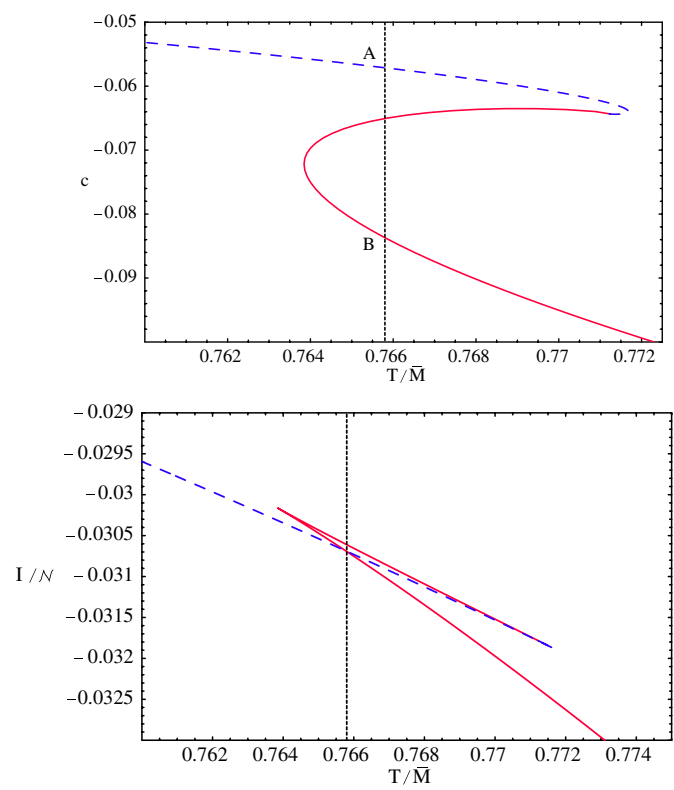

FIG. 2 (color online). Quark condensate and free energy for a $D 7$ in a $D 3$ background. The dashed (solid) curves correspond to the Minkowski (black hole) embeddings. The dotted vertical line indicates the precise temperature of the phase transition. 
if this could be compared to the strong coupling limit of a perturbative calculation. The same observations apply for other thermodynamic quantities such as, e.g., the entropy density $S=-\partial_{T} F$. We remind the reader that the background geometry makes the leading contribution to the free-energy density [15]

$$
F \sim N_{c}^{2} T^{p+1} g_{\text {eff }}(T)^{2(p-3) /(5-p)},
$$

which corresponds to that coming from the gluons and adjoint matter. Of course, for $p=3$, the effective coupling factor is absent and this bulk contribution differs from the weak coupling result by only a factor of $3 / 4$ [16].

A striking feature of this new phase transition is found in the spectrum of mesons (i.e., of quark-antiquark bound states). The latter correspond to excitations supported on the probe branes [12]. Fluctuation analyses [17] reveal that, for the Minkowski embeddings, the meson spectrum is discrete and possesses a mass gap, while for the black hole embeddings, it is continuous and gapless. This implies that, in a large class of gauge theories with fundamental matter, quark-antiquark bound states survive the deconfining phase transition for the gluonic degrees of freedom provided $\bar{M} \gtrsim T_{D}$, where $\bar{M}$ is the typical mesonic scale. This is potentially interesting in connection with QCD, since in QCD heavy quark mesonic bound states with $\bar{M} \gg T_{D} \sim 175 \mathrm{MeV}$ certainly exist. One generic feature of the low-lying mesons in the class of theories discussed here is that they are extremely deeply bound at strong coupling [11,12], as is apparent from Eq. (11). It is intriguing that the mesonic states claimed to explain certain features (such as the entropy density) of the strongly coupled quark-gluon plasma formed at RHIC are also deeply bound [18]. It would be remarkable to establish a precise relationship between the two.

Of particular interest are thermal phase transitions in gauge theories with spontaneously broken chiral symmetries (at zero temperature). Here the question is when the chiral symmetry is restored, as was recently studied in Ref. [19] for the $D 4 / D 8 / \bar{D} 8$ model of Ref. [20]. With the insights of the present Letter, we see that their results indicate that chiral symmetry is restored as a separate phase transition if $\bar{M}>T_{D}$, where the meson scale is now defined in terms of the constituent quark mass. However, self-similar behavior is absent because the probe brane topology is different. The $D 8 / \overline{D 8}$ probes split into two disconnected pieces upon falling into the horizon. In the gauge theory, this difference in topologies is reflected in the fact that the fundamental matter lives on a pair of defects, rather than a single defect as considered here.

Several of the conclusions reached in the $N_{c}, \lambda \rightarrow \infty$ approximation are likely to be affected once finite- $N_{c}$ and finite- $\lambda$ corrections are included. For example, at large but finite $N_{c}$, the black hole will Hawking-radiate and the brane probe will experience a thermal bath at a temperature determined by the local acceleration. Note that this effect is of order $1 / N_{c}^{2}$ and, therefore, subleading with respect to the order- $N_{f} / N_{c}$ contributions of the brane probes. Finite 't Hooft coupling corrections correspond to higherderivative corrections to both the supergravity and $D$-brane actions. These may spoil the self-similar behavior. We will return to these and other issues in Ref. [17].

We thank V. Frolov for a conversation and for sharing Ref. [7] with us prior to publication and A. Starinets and P. Kovtun for discussions. Research at the Perimeter Institute is supported in part by funds from NSERC of Canada and MEDT of Ontario. We also acknowledge support from NSF Grant No. PHY-0244764 (D. M.) and an NSERC Discovery grant (R.C.M.). D.M. and R.C.M. also thank the KITP for hospitality in the early stages of this project. Research at the KITP was supported in part by the NSF under Grant No. PHY99-07949.

[1] P. Kovtun, D. T. Son, and A. O. Starinets, J. High Energy Phys. 10 (2003) 064; Phys. Rev. Lett. 94, 111601 (2005); A. Buchel and J. T. Liu, Phys. Rev. Lett. 93, 090602 (2004).

[2] E. Shuryak, Prog. Part. Nucl. Phys. 53, 273 (2004).

[3] A. Karch and L. Randall, J. High Energy Phys. 06 (2001) 063; A. Karch and E. Katz, J. High Energy Phys. 06 (2002) 043.

[4] E. Witten, Adv. Theor. Math. Phys. 2, 505 (1998).

[5] M. Kruczenski et al., J. High Energy Phys. 05 (2004) 041.

[6] J. Babington et al., Phys. Rev. D 69, 066007 (2004); I. Kirsch, Fortschr. Phys. 52, 727 (2004); R. Apreda et al., Phys. Rev. D 71, 126002 (2005); K. Ghoroku et al., Phys. Rev. D 71, 106002 (2005).

[7] V. P. Frolov, Phys. Rev. D 74, 044006 (2006).

[8] V.P. Frolov, A.L. Larsen, and M. Christensen, Phys. Rev. D 59, 125008 (1999); M. Christensen, V. P. Frolov, and A. L. Larsen, Phys. Rev. D 58, 085008 (1998).

[9] We assume $n>1$. For $n=1$, the second is $c \log \rho / \rho$.

[10] N. Itzhaki et al., Phys. Rev. D 58, 046004 (1998).

[11] R. C. Myers and R. M. Thomson, hep-th/0605017.

[12] M. Kruczenski et al., J. High Energy Phys. 07 (2003) 049.

[13] M. Henningson and K. Skenderis, J. High Energy Phys. 07 (1998) 023; Fortschr. Phys. 48, 125 (2000); V. Balasubramanian and P. Kraus, Commun. Math. Phys. 208, 413 (1999); R. Emparan et al., Phys. Rev. D 60, 104001 (1999); C. R. Graham, math.dg/9909042.

[14] A. Karch, A. O'Bannon, and K. Skenderis, J. High Energy Phys. 04 (2006) 015.

[15] A. W. Peet and J. Polchinski, Phys. Rev. D 59, 065011 (1999).

[16] S. S. Gubser, I. R. Klebanov, and A. W. Peet, Phys. Rev. D 54, 3915 (1996).

[17] D. Mateos, R.C. Myers, and R. M. Thomson (to be published).

[18] G. E. Brown, B. A. Gelman, and M. Rho, Phys. Rev. Lett. 96, 132301 (2006).

[19] O. Aharony, J. Sonnenschein, and S. Yankielowicz, hepth/0604161; A. Parnachev and D. A. Sahakyan, hep-th/ 0604173.

[20] T. Sakai and S. Sugimoto, Prog. Theor. Phys. 113, 843 (2005). 